\title{
THE EFFECT OF A SUBMERGED BARRIER ON THE NATURAL FREQUENCIES AND RADIATION DAMPING OF A SHALLOW BASIN CONNECTED TO OPEN WATER
}

\author{
E. O. TUCK \\ (Received 1 November 1979) \\ (Revised 17 January 1980)
}

\begin{abstract}
A theory is provided for the natural seiching frequencies and radiative decay rates for a shallow-water basin whose connection to open water is restricted by a submerged wall or reef. The transition from an essentially-open basin to a closed basin, as the aperture reduces to zero, is discussed using a matching procedure. Graphs of frequencies and damping factors as functions of aperture size are obtained for idealized two-dimensional shelf configurations, involving a constant-depth shallow basin connected to constant-depth, but not necessarily shallow, open water.
\end{abstract}

\section{Introduction}

The natural two-dimensional seiching frequencies $\sigma$ of a closed shallow-water basin of length $L$ and uniform depth $h_{1} \ll L$ are given by

$$
\frac{\sigma L}{\sqrt{\left(g h_{1}\right)}}=n \pi, \quad n=1,2,3, \ldots .
$$

These natural modes possess antinodes of surface elevation or nodes of horizontal velocity at the two end walls of the basin, and are damped only by friction, here neglected.

If one end of the basin is open to a much larger body of water, it is possible to establish a different set of natural modes satisfying

$$
\frac{\sigma L}{\sqrt{\left(g h_{1}\right)}}=\left(n+\frac{1}{2}\right) \pi, \quad n=0,1,2,3, \ldots .
$$

These modes still possess an antinode of surface elevation at the closed end, but now possess a node at the open end. Such modes can be thought of as analogous to those 
for an open organ pipe in acoustics, and also have zero damping if friction is neglected.

The problem treated in this paper is that corresponding to a transition between these two extreme cases, in which the connection between the basin and the open water is through an underwater opening, controlled by a submerged vertical wall, leaving a gap of size $a$ between its top edge and the mean free surface. When the wall breaks the surface (that is, $a=0$ ) the basin is closed and the natural frequencies are given by (1.1). On the other hand, for any non-zero value of $a$ (especially in the absence of a wall, $a=h_{1}$ ), the open basin frequencies (1.2) are obtained in the formal limit of extreme shallowness, as $h_{1} / L \rightarrow 0$. Our aim is to display the transition between these extremes, as we reduce the gap size from $a=h_{1}$ (no wall) to $a=0$ (no gap). At the same time, we compute the damping, due to radiation of wave energy to infinity through the gap : this is zero only at $a=0$, for fixed non-zero $h_{1} / L$

The results show as expected that the natural frequencies change continuously from values close to the open-basin values (1.2) to the closed-basin values (1.1), the mode numbers $n=1,2,3, \ldots$ being fixed during this transition. Thus, in general, for any fixed mode $n$, the frequency decreases as the gap size $a$ decreases.

This is also true of the lowest-order open-basin mode $n=0$, in spite of the fact that formally there exists nō corresponding closed mode, and the frequency of this mode tends to zero as $a \rightarrow 0$. The limiting flow inside the basin for this mode corresponds to a uniform low-frequency rising and falling of the free surface. This is a Helmholtz resonator [5] in which the basin simply serves as a potential-energy store, inertia for the oscillations being supplied by the fluid moving through the vanishingly-small gap.

The rate at which the closed-basin result is approached as $a \rightarrow 0$ is extremely slow : for example, corresponding to the reciprocal of the logarithm of $a$. Thus, as $a$ decreases, the natural frequency (and to a lesser extent the damping) remains almost constant until $a / h_{1}$ takes quite unrealistically small values, of the order of 0.01 or less. Thus, in practice, one should not expect to observe a continuous transition to the closed-basin values in any oceanographical or experimental situation. Instead, neglected phenomena such as non-linearity and surface tension may cause a sudden jump to a fully closed basin as soon as $a / h_{1}$ reaches a critical low value; above that value, to all intents and purposes the basin is open.

In fact, the present theoretical investigation was initiated to explain observations [1] of natural seiching periods of the order of $30 \mathrm{~min}$ on the continental shelf off the coast of Western Australia, at a point where a submerged reef is a prominent feature at a point approximately $5.5 \mathrm{~km}$ off shore. Observational evidence and results of model experiments are in general agreement with the present theory and will be reported elsewhere [8].

In the present paper the analysis is developed for free oscillations only, using linearized water-wave theory. Thus we seek solutions whose time dependence is of 
the form $e^{-i \sigma t}$, where $\sigma$ is a complex number whose real part gives the radian frequency and whose imaginary part is negative, corresponding to decaying or damped oscillations. We obtain a non-linear dispersion relation from which we can determine the spectrum of values of $\sigma$.

The basin itself is always assumed to be shallow, that is, $h_{1} / L$ is assumed small. However, we allow either a shallow or a deep body of open water. That is, if $h_{2}$ is the depth of water beyond the wall, we place no restrictions on the ratio $\mu=h_{2} / h_{1}$.

As an intermediate step in the solution of this problem, it is necessary to solve a local potential-flow problem in the immediate vicinity of the wall, and conformal mapping methods are used here for this purpose, for a representative idealized wall geometry. However, in principle such a computation can be performed for any type of transition, the required output parameter being a single "blockage coefficient" $C$, which characterizes the transition as seen in its own far field [7]. Once this quantity $C$ is known, the actual geometrical details of the wall and transition are of no further significance for the determination of the complex frequencies $\sigma$.

The present analysis may easily be extended to forced oscillations, for example, those produced by allowing given incident wave energy from the open water, or by inserting a wave maker at the closed end, and so on. In such cases one is interested in the steady-state sinusoidal response at a frequency $\sigma_{F}$ that is necessarily real. The response of the basin is then expected to be greatest at frequencies $\sigma_{F}$ close to the natural frequencies $\mathscr{R} \sigma$ predicted in the present paper, with a resonant amplitude inversely dependent on the damping $\mathscr{I} \sigma$.

This type of forced-oscillation problem is analogous to the "harbour resonance" problem, discussed by many authors. In particular, the "harbour paradox" [3], which refers to a tendency (at least for the Helmholtz mode $n=0$ ) for the resonant response to increase as a harbour opening is closed off in the horizontal plane, will have its counterpart in the present problem with respect to vertical-plane closures.

\section{Problem formulation and shallow water approximation}

The exact problem of interest is as sketched in Fig. 1. We have to solve Laplace's equation

$$
\Phi_{x x}+\Phi_{y y}=0
$$

for the velocity potential $\Phi(x, y, t)$ in a region of the $(x, y)$ plane bounded by impermeable surfaces, a linearized free surface and a boundary at $x=+\infty$. The impermeable surfaces include the left end

$$
\frac{\partial \Phi}{\partial x}=0 \quad \text { on } x=-L, \quad-h_{1}<y<0,
$$




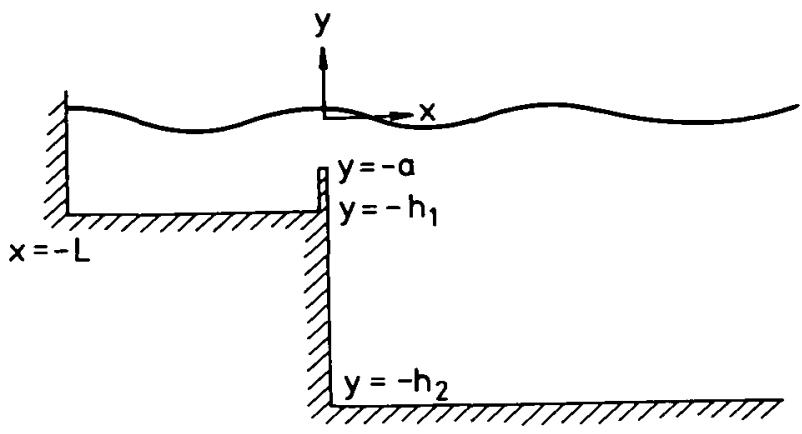

Fig. 1. Sketch of flow geometry.

the bottoms

$$
\begin{gathered}
\frac{\partial \Phi}{\partial y}=0 \quad \text { on } y=-h_{1}, \quad-L<x<0, \\
\text { and } y=-h_{2}, \quad x>0,
\end{gathered}
$$

and the wall and step face

$$
\begin{aligned}
\frac{\partial \Phi}{\partial x}=0 \quad & \text { on } x=0_{-}^{\circ}, \quad-h_{1}<y<-a, \\
& \text { and } x=0_{+}, \quad-h_{2}<y<-a .
\end{aligned}
$$

Thus $h_{1}$ and $h_{2}$ are the two water depths of interest, and the wall leaves a gap of size $a$ beneath the free surface.

The linearized free-surface condition is ([9], page 470$)$

$$
g \frac{\partial \Phi}{\partial z}+\frac{\partial^{2} \Phi}{\partial t^{2}}=0, \quad y=0, \quad x>-L
$$

where $g$ is the acceleration of gravity, and applies to flows in which the free-surface displacement

$$
y=\eta(x, t)=-\frac{1}{g} \Phi_{t}(x, 0, t)
$$

is everywhere small compared to all the length scales. The boundary condition at infinity, $x \rightarrow+\infty$, is that any wave present must be outgoing, that is, travelling in the $+x$ direction. We postpone an exact statement of this condition.

We seek solutions of the form

$$
\Phi(x, y, t)=\mathscr{R} \phi(x, y) e^{-i \sigma t} .
$$


If $\sigma$ is real, this means that we seek solutions that are sinusoidal with respect to time, with period $2 \pi / \sigma$. However, we shall find that $\sigma=\sigma_{1}+i \sigma_{2}$ is complex, in which case such a solution either grows $\left(\sigma_{2}>0\right)$ or decays $\left(\sigma_{2}<0\right)$ with time. Equations (2.1) $-(2.4)$ still hold with $\Phi$ replaced by $\phi$, and (2.5) becomes

$$
\phi_{y}-v \phi=0, \quad v=\sigma^{2} / g, \quad \text { on } y=0, \quad x>-L .
$$

The far-field condition can now be stated explicitly, namely

$$
\phi \rightarrow e^{i k_{2} x} \frac{\cosh \left[k_{2}\left(y+h_{2}\right)\right]}{\cosh \left(k_{2} h_{2}\right)} \text { as } x \rightarrow+\infty .
$$

The expression (2.8) satisfies Laplace's equation, and the bottom condition (2.3), and satisfies (2.7) if $k_{2}$ is a solution of the dispersion equation

$$
\nu=k_{2} \tanh \left(k_{2} h_{2}\right) \text {. }
$$

The "outgoing" nature of the far-field is guaranteed by the choice $e^{i k_{2} x}$ rather than $e^{-i k_{2} x}$, in combination with the $e^{-i \sigma t}$ time dependence. The solution with the property (2.8) has been normalized to have a prescribed free-surface amplitude (unity for $\phi$ and $i \sigma / g$ for $\eta$ ) at infinity.

The boundary-value problem so formulated for $\phi$ can be expected to possess a unique solution, and can in principle be solved by numerical means (for example, as in [2]) for arbitrary values of the length scales $h_{1}, h_{2}, L, a$ and $v^{-1}$. In this paper we consider only shallow inner basins, with $h_{1} \ll L$, and waves which are capable of resonating in the inner basin if closed, that is, $v^{-1}$ is comparable to $L$ and therefore large compared to $h_{1}$. Thus, at least in the inner basin (not too close to the transition at $x=0$ ), we can make the shallow-water approximation that $\phi$ varies slowly with respect to $y$.

For example, upon Taylor expansion about $y=0$, we have

$$
\begin{aligned}
\phi(x, y) & =\phi(x, 0)+y \phi_{y}(x, 0)+\frac{1}{2} y^{2} \phi_{y y}(x, 0)+\ldots \\
& =\phi(x, 0)+v y \phi(x, 0)-\frac{1}{2} y^{2} \phi_{x x}(x, 0)+\ldots
\end{aligned}
$$

which satisfies the bottom condition (2.3) if

$$
h \phi_{x x}+v \phi=0
$$

with $h=h_{1}$. More generally, if the bottom is of variable depth $h(x)$,

$$
\frac{\partial}{\partial x}\left(h(x) \phi_{x}\right)+v \phi=0 .
$$

This equation does not hold in the immediate neighbourhood $x=O\left(h_{1}\right)$ of the depth transition, since there one can expect significant upward velocity components. However, when $x$ and $y$ are both $O\left(h_{1}\right)$, where $v^{-1} \gg h_{1}$, it is clear (for example, by 
stretching $x$ and $y$ by the factor $1 / h_{1}$ ) that the free-surface condition (2.7) simplifies locally to the impermeable-surface condition

$$
\phi_{y}=0 \quad \text { on } y=0, \quad x=O\left(h_{1}\right)
$$

That is, sharp depth transitions are not describable by the one-dimensional shallowwater equation (2.12), but require solution of the full two-dimensional Laplace equation (2.1), with the simplifying feature that the free surface may be replaced by an impermeable lid. A more-detailed asymptotic analysis as $v h_{1} \rightarrow 0$ (for example, as in [5], [7]) indicates that the error in all of these approximations is quadratic in the ratio of length scales, that is, is a factor $1+O\left(v h_{1}\right)^{2}$.

Equation (2.11) may be solved immediately with $h=h_{1}=$ constant, giving

$$
\phi=A \cos \left[k_{1}(x+L)\right]
$$

for some constant $A$ to be determined, where

$$
k_{1}=\sigma / \sqrt{ }\left(g h_{1}\right)
$$

This solution possesses an antinode at the closed end $x=-L$, so satisfying (2.2). If $a=0$ (that is, the wall completely closes off the basin at $x=0$ ), we must have $\phi_{x}=0$ at $x=0$, so that $\sin \left(k_{1} L\right)=0$, that is,

$$
k_{1} L=\pi, 2 \pi, 3 \pi, \ldots
$$

This corresponds to occurrence of an antinode at $x=0$ as well. On the other hand, if the flow is such that a node occurs at $x=0$ (that is, $\phi=0$ at $x=0$ ), we must have $\cos \left(k_{1} L\right)=0$, that is,

$$
k_{1} L=\frac{\pi}{2}, \frac{3 \pi}{2}, \frac{5 \pi}{2}, \ldots
$$

This may be described as an "open" basin situation.

In fact, neither of these situations occurs for the geometry of Fig. 1, and hence neither (2.16) nor (2.17) gives the correct values of $k_{1}$ and hence, by (2.15), of the frequency $\sigma$. The true situation with $a \neq 0$ is between these extremes, and our aim is to determine the possible values of $k_{1} L$, for the actual geometrical situation of Fig. 1 .

Note that the limiting values (2.16) and (2.17) of $k_{1}$, and hence of $\sigma$, are real. However, in the actual problem we cannot expect this to be true, since the connection to infinity through the non-zero gap at $x=0$ implies a leakage of energy, and hence a radiation damping. Thus we expect a negative imaginary part $\sigma_{2}$ to the quantity $\sigma$, corresponding to decay of wave amplitude with respect to time. 


\section{Shallow-water transitions}

We first assume that not only $h_{1}$ but also $h_{2}$ is a shallow depth, compared to $L$ and $v^{-1}$. Then the shallow-water equation (2.11) also applies with $h=h_{1}, x>0$. The appropriate solution in that region is

$$
\phi=e^{k_{2 x} x},
$$

where $k_{2}=\sigma / \sqrt{ }\left(g h_{2}\right)$. This is nothing more than the limit as $k_{2} h_{2} \rightarrow 0$ of $(2.8)$ and (2.9), as expected, and represents a pure outgoing wave. The solution (3.1) applies for $x>0$, but only so long as $x \gg O\left(h_{2}\right)$.

When $x=O\left(h_{1}\right)=O\left(h_{2}\right)$ we must solve a local-flow problem encompassing the complete depth transition and wall, but with a rigid lid as a free surface. The boundary conditions at "infinity" for this local-flow problem are obtained by matching with the behaviour of the shallow-water solutions as $x \rightarrow 0$.

Thus, as $x \rightarrow 0_{-}$, the shelf solution (2.14) reduces to

$$
\phi \rightarrow\left[A \cos \left(k_{1} L\right)\right]+x\left[-k_{1} A \sin \left(k_{1} L\right)\right],
$$

which is interpretable as a uniform stream of speed

$$
U_{1}=-k_{1} A \sin \left(k_{1} L\right)
$$

together with an additive constant $A \cos \left(k_{1} L\right)$. The condition (3.2) is now used as the left-hand-infinity condition for the local flow through the gap, that is, as $x \rightarrow-\infty$. Similarly, as $x \rightarrow 0_{+}$in the outer solution (3.1), we have

$$
\phi \rightarrow 1+i k_{2} x,
$$

which corresponds to a uniform stream of magnitude

$$
U_{2}=i k_{2},
$$

and we use (3.4) as the right-hand-infinity condition for the local flow through the gap.

This completes specification of the local-flow problem in the $(x, y)$ plane for $x, y=O\left(h_{1}\right)=O\left(h_{2}\right)$. This problem can be solved easily by conformal mapping, and the complete solution is provided in Appendix $A$. It is convenient to normalize in such a way that the net flux through the gap is of unit value, and the additive constant is antisymmetric between the two infinities. That is, we write

$$
\phi(x, y)=m \phi^{*}(x, y)+\frac{1}{2}+\frac{1}{2} A \cos \left(k_{1} L\right),
$$

where

$$
m=U_{1} h_{1}=U_{2} h_{2}
$$

is the net volume flux and $\phi^{*}(x, y)$ is a normalized potential satisfying 


$$
\phi^{*} \rightarrow \begin{cases}\frac{x}{h_{1}}-C & \text { as } x \rightarrow-\infty, \\ \frac{x}{h_{2}}+C & \text { as } x \rightarrow+\infty,\end{cases}
$$

for some constant $C$. Then both boundary conditions (3.2) and (3.4) are satisfied providing

$$
1-A \cos \left(k_{1} L\right)=2 m C .
$$

The non-dimensional quantity $C=C\left(h_{2} / h_{1}, a / h_{1}\right)$ is a unique property of the geometry of the transition, which can be called the "blockage coefficient". It is determined in Appendix $A$ and plotted in Fig. 9, and may now be treated as known. Now the flux conservation law (3.7) implies that

$$
m=-k_{1} h_{1} A \sin \left(k_{1} L\right)=i k_{2} h_{2},
$$

that is,

$$
m=i k_{2} h_{2}
$$

and

$$
A=-i\left(\frac{h_{2}}{h_{1}}\right)^{\frac{1}{2}} \operatorname{cosec}\left(k_{1} L\right)
$$

are both known, given $k_{1}$. On substitution into (3.9), we have

$$
\cot \left(k_{1} L\right)=2 C k_{1} h_{1}+i\left(\frac{h_{1}}{h_{2}}\right)^{\frac{1}{2}} \text {. }
$$

Equation (3.12) is the main product of our efforts, namely a dispersion relation that determines the wave-number $k_{1}$ and hence the complex frequency $\sigma=\sigma_{1}+i \sigma_{2}$. In fact, under most circumstances $C=O(1)$ and, since $k_{1} h_{1} \ll 1$, the term $2 C k_{1} h_{1}$ on the right can be neglected. Thus, for $C=O(1)$, we have the set of eigenvalues with $n=1,2,3, \ldots$,

$$
k_{1} L=\left\{\begin{array}{l}
\left(n-\frac{1}{2}\right) \pi-\frac{1}{2} i \log \left(\frac{\mu^{\frac{1}{2}}+1}{\mu^{\frac{1}{2}}-1}\right), \quad \mu>1, \\
n \pi-\frac{1}{2} i \log \left(\frac{1+\mu^{\frac{1}{2}}}{1-\mu^{\frac{1}{2}}}\right), \quad \mu<1,
\end{array}\right. \text {. }
$$

where

$$
\mu=h_{2} / h_{1} \text {. }
$$

The imaginary part of $k_{1}$ is negative, indicating decay due to radiative damping. The real part gives the natural frequencies, which correspond to closed basins as in (2.16) if $h_{2}<h_{1}(\mu<1)$ and open basins as in (2.17) if $h_{1}<h_{2}(\mu>1)$. These modes are, however, strongly damped unless $\mu$ is either very small or very large. In particular, 
the cross-over at $\mu=1$ is through an "infinitely-damped" flow, that is, no flow at all of the type being considered here exists when there is no depth transition.

From now on we confine attention to the case $\mu>1$ of a transition to deeper outer water. In particular, as $\mu \rightarrow \infty$, we recover the open-basin frequencies (2.17), and the damping vanishes. We now ask what is the effect of the (small?) term $2 C k_{1} h_{1}$ in (3.12), especially when the geometry is such that $C$ takes relatively large values.

In fact, as is shown in Appendix A, $C \rightarrow+\infty$ as $a / h_{1} \rightarrow 0$. That is, as we increase the wall height until there is no gap at all, and the inner basin is completely closed off, we have $2 C k_{1} h_{1} \rightarrow+\infty$, and hence $\cot \left(k_{1} L\right) \rightarrow+\infty$, which corresponds to the closed-basin frequencies (2.16). Thus (3.12) provides for $\mu>1$ (especially for large $\mu$ ), a natural interpolation between the open and closed basins, as the wall height is increased.

However, $C \rightarrow \infty$ rather slowly (logarithmically) as $a / h_{1} \rightarrow 0$, as is seen from Fig. 9 and equation (A16). Hence the basin remains effectively open irrespective of the height of the wall, until the gap has almost been reduced to zero, when it converts rapidly into a closed basin.

\section{Non-shallow outer depth}

For any fixed but small value of $h_{1} / L$, the results of Section 3 necessarily become suspect as $\mu \rightarrow \infty$, since in that limit the outer water can no longer be assumed to be shallow. If we are interested in large values of $\mu$, that is, $h_{2} \gg h_{1}$, we must relax the assumption that shallow-water theory can be used for $x>0$.

At the same time, however, there is a new simplification to the geometry of the transition region as $\mu \rightarrow \infty$, since the local transition must now be considered to take place between a finite depth $h_{1}$ for $x<0$ and an apparently-infinite depth for $x>0$, as sketched in Fig. 10. The flow emerging from the gap now appears to an observer in $x=O\left(h_{2}\right) \gg h_{1}$ like that due to an isolated source.

Thus, although the shallow-water outer solution (3.1) is no longer available to us, we can still write down a suitable approximate solution in $x>0$, in the form of a source located at the origin in water of finite depth $h_{2}$. The following is a suitable potential for our purpose; it is constructed by modifying well-known results, for example, those given by Wehausen and Laitone ([9], page 483) and Macaskill [2]. We write

$$
\phi(x, y)=\cos \left(k_{2} x\right) \frac{\cosh \left[k_{2}\left(y+h_{2}\right)\right]}{\cosh \left(k_{2} h_{2}\right)}+\frac{2}{\pi} m\left\{\log \frac{r}{h_{2}}+\log \frac{r^{\prime}}{h_{2}}+I(x, y)\right\},
$$

where

$$
r=\sqrt{ }\left(x^{2}+y^{2}\right), \quad r^{\prime}=\sqrt{ }\left(x^{2}+\left(y+2 h_{2}\right)^{2}\right), \quad m=\frac{i}{2 k_{2}}\left[v+h_{2}\left(k_{2}^{2}-v^{2}\right)\right],
$$


and

$$
\begin{aligned}
I(x, y)=\int_{0}^{\infty} d k \frac{e^{-k h_{2}}}{k}\{ & -2+\cos k x \cosh k\left(y+h_{2}\right) \\
\times & {\left.\left[1-\frac{(v+k) \cosh k h_{2}}{k \sinh k h_{2}-v \cosh k h_{2}}\right]\right\} . }
\end{aligned}
$$

The potential $\phi(x, y)$ of (4.1) satisfies Laplace's equation, has vanishing vertical velocity on $y=-h_{2}$, satisfies the free surface condition (2.7) if (2.9) holds, and satisfies (2.8) as $x \rightarrow+\infty$. Note that a Cauchy principal value interpretation is to be given to the integral in (4.3) and that, since $m$ is pure imaginary, only the first term in (4.1) is real, if $\sigma$ happens to be real.

In order to match with the flow emerging from the gap where $r=O\left(h_{1}\right) \ll h_{2}$, we let $x, y \rightarrow 0$ in (4.1) and find

$$
\phi \rightarrow 1+\frac{2}{\pi} m\left[\log \frac{2 r}{h_{2}}+\mathbf{I}\left(v h_{2}\right)\right]
$$

where

$$
\begin{aligned}
\mathbf{I}\left(v h_{2}\right) & =I(0,0) \\
& =\int_{0}^{\infty} \frac{d k}{k} e^{-k h_{2}}\left\{-2+\cosh k h_{2}-\frac{(v+k) \cosh ^{2} k h_{2}}{k \sinh k h_{2}-v \cosh k h_{2}}\right\} .
\end{aligned}
$$

Setting $H=v h_{2}$, we have

$$
\mathbf{I}(H)=\int_{0}^{\infty} \frac{d t e^{-t}}{t}\left\{-2+\cosh t-\frac{(H+t) \cosh ^{2} t}{t \sinh t-H \cosh t}\right\}
$$

a function that may be computed easily. Figure 2 shows a plot of $\mathbf{I}(H)$, evaluated directly from (4.6) using the trapezoidal rule, combined with Monacella's [4] method of ignoring the singularity, and assuming for the present that $H$ is real.

The above results all simplify considerably if the outer depth $h_{2}$ is actually infinite, or if $H=v h_{2}$ is large, in which case

$$
\mathbf{I}(H) \rightarrow \log (H / 2)+\gamma,
$$

where $\gamma=0.5772 \ldots$ is Euler's constant, and

$$
\phi \rightarrow 1+\frac{i}{\pi}(\log v r+\gamma)
$$

which is independent of $h_{2}$ as required. Figure 2 shows the infinte-depth asymptote (4.7) dashed. At the other extreme, in the shallow-water limit as $H \rightarrow 0$, we have $\mathbf{I}(0)=\log (\pi / 2)=0.4516 \ldots$. 


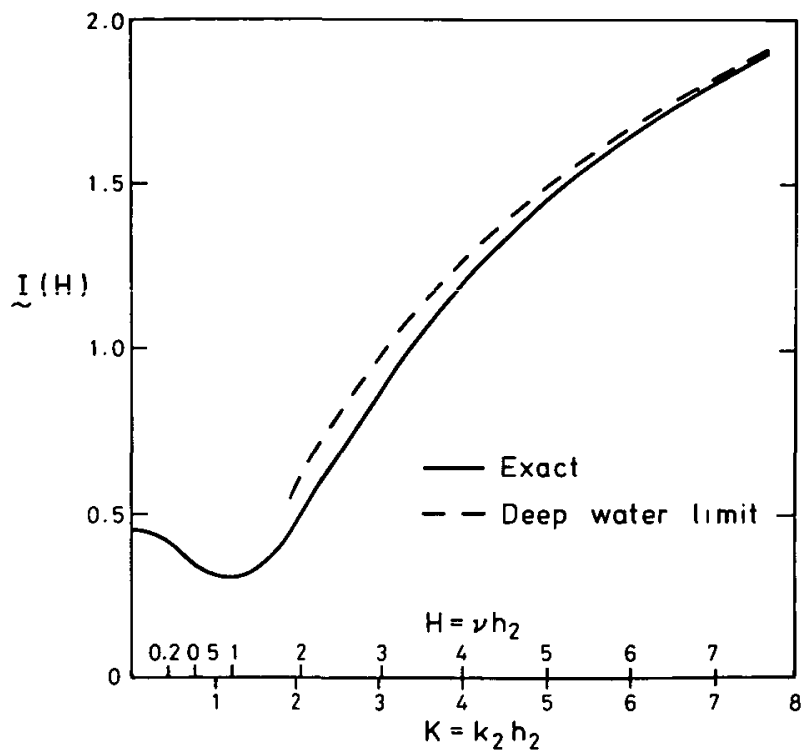

Fig. 2. Function $\mathbf{I}(H)$ for finite outer depths. Deep-water approximation dashed.

We are now in a position to match with the local flow through the gap using (4.4) as the boundary condition as $x \rightarrow+\infty$ for such a flow. Thus we write as the gapregion flow (compare with (3.6))

$$
\phi(x, y)=m \phi^{* *}(x, y)+A \cos k_{1} L,
$$

where $\phi^{* *}$ is a canonical potential for a transition as in Fig. 10 from depth $h_{1}$ to infinte depth, satisfying

$$
\phi^{* *} \rightarrow\left\{\begin{array}{l}
\frac{x}{h_{1}} \text { as } x \rightarrow-\infty \\
\frac{2}{\pi}\left(\log \frac{r}{h_{1}}+C_{\infty}\right) \text { as } x \rightarrow+\infty,
\end{array}\right.
$$

where $C_{\infty}=C_{\infty}\left(a / h_{1}\right)$ is a known constant, given by (A24). Then the boundary condition (3.2) is satisfied if, as in Section 3,

$$
m=-k_{1} h_{1} A \sin \left(k_{1} L\right)
$$

and the new boundary condition (4.4) is satisfied if

$$
A \cos \left(k_{1} L\right)=1-\frac{2}{\pi} m\left[\log \frac{h_{2}}{2 h_{1}}-\mathbf{I}+C_{\infty}\right] .
$$

Upon the elimination of $A$ between (4.11) and (4.12), and use of (4.2) for $m$, we have the dispersion relation 


$$
\cot \left(k_{1} L\right)=\frac{2}{\pi} k_{1} h_{1}\left[\log \frac{h_{2}}{2 h_{1}}-\mathbf{I}\left(v h_{2}\right)+C_{\infty}\left(a / h_{1}\right)\right]+\frac{2 i k_{1} k_{2} h_{1}}{v+h_{2}\left(k_{2}^{2}-v^{2}\right)} .
$$

Equation (4.13) has a similar structure to that of (3.12). The cotangent of $k_{1} L$ is again given by a real quantity plus a positive imaginary quantity, and again we can expect damped solutions, with $k_{1}<0$. Again, because $k_{1} h_{1} \ll 1$, the real part is normally small, but now under such circumstances that so is the imaginary part. Thus, except where the appropriate coefficients take large values, the cotangent is small and the open-basin natural frequencies (2.17) are good approximations. This simply reflects the fact that in the absence of special blocking features, a transition from shallow to deep water appears on the shallow side as that for an open basin.

The special case of an infinitely-deep outer basin, or one in which $v h_{2} \gg 1$, is of interest, and then, using (4.7), equation (4.13) reduces to

$$
\cot \left(k_{1} L\right)=\frac{2}{\pi} k_{1} h_{1}\left[C_{\infty}-\log v h_{1}-\gamma+i \pi\right] .
$$

In the opposite limit of a shallow outer basin, equations (4.13) and (3.12) give identical results. That is, the common limit of (3.12) as $\mu \rightarrow \infty$, and (4.13) as $v h_{2} \rightarrow 0$, is

$$
\cot \left(k_{1} L\right)=\frac{2}{\pi} k_{1} h_{1}\left[\log \frac{\mu}{\pi}+C_{\infty}\right]+i \mu^{-\frac{1}{2}}
$$

\section{Computed results}

Our task is to solve the dispersion relation (3.12) or (4.13) for the complex quantity

$$
s=k_{1} L=s_{1}+i s_{2} .
$$

Both equations are parameterized by $\mu=h_{2} / h_{1}, a / h_{1}$ and

$$
\varepsilon=h_{1} / L
$$

Thus (3.12) can be written as

$$
0=f(s)=\cot s-2 s \varepsilon C\left(\mu, \frac{a}{h_{1}}\right)-i \mu^{-\frac{1}{2}},
$$

and (4.13) as

$$
\begin{gathered}
0=f(s)=\cot s-\frac{2}{\pi} s \varepsilon\left[\log \frac{1}{2} \mu-\mathbf{I}\left(s^{2} \varepsilon^{2} \mu\right)+C_{\infty}\left(\frac{a}{h_{1}}\right)\right] \\
-\frac{2 i s \varepsilon K}{s^{2} \varepsilon^{2} \mu+K^{2}-s^{4} \varepsilon^{4} \mu^{2}},
\end{gathered}
$$


where $K=k_{2} h_{2}=K(s)$ is determined from (2.9), that is,

$$
H=v h_{2}=s^{2} \varepsilon^{2} \mu=K \operatorname{tank} K .
$$

The infinite-outer-depth limit (4.14) can be written

$$
0=f(s)=\cot s-\frac{2}{\pi} s \varepsilon\left[C_{\infty}-\log \left(s^{2} \varepsilon^{2} \mu\right)-\gamma+i \pi\right] .
$$

We use Newton's method to solve these equations for $s$. That is, given a starting guess $s=s_{0}$, we improve it by repeated use of

$$
s=s_{0}-\frac{f\left(s_{0}\right)}{f^{\prime}\left(s_{0}\right)},
$$

where the function $f(s)$ and its derivative $f^{\prime}(s)$ are obtained from (5.3), (5.4) or (5.6). There are infinitely many solutions $s$, and the Newton iteration will converge (if at all) to a mode determined by the initial starting point. It is found that starting with the real open-basin values (2.17) enables convergence in most cases, although for some higher modes a more-accurate initial choice needs to be found by trial and error.

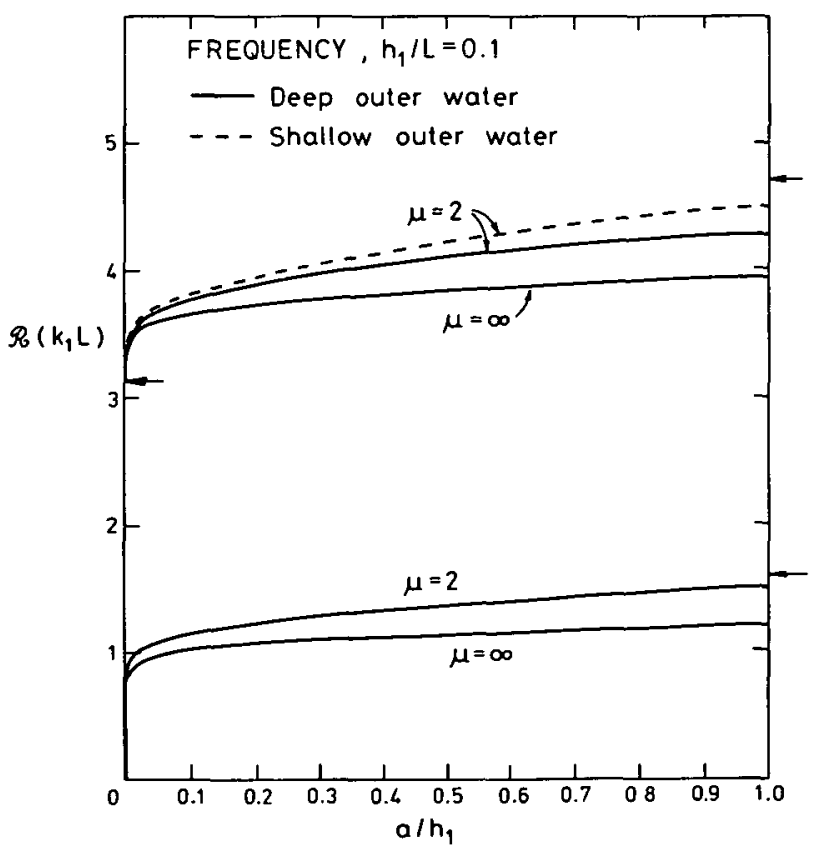

Fig. 3. Real part of frequency at $\varepsilon=h_{1} / L=0.1$, plotted against $a / h_{1}$ for $\mu=2$ and $\mu=\infty$. For the second mode at $\mu=2$, the dashed curve assumes shallow water in the open water and the solid curve finite depth.

These curves are indistinguishable for the first mode. 


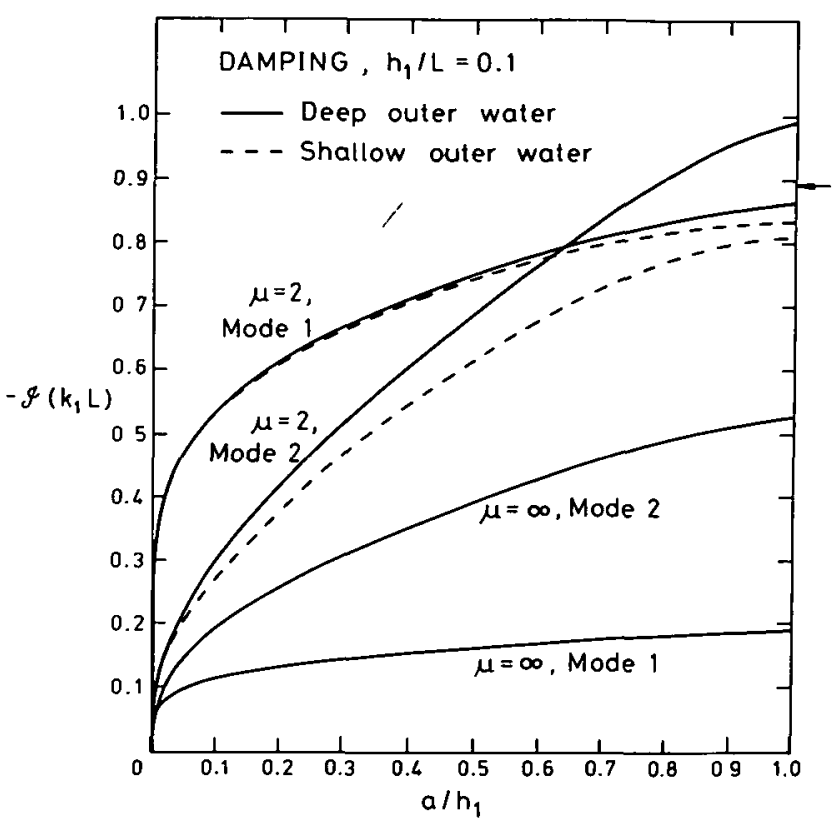

Fig. 4. Negative of imaginary part of frequency (that is, damping factor) at $\varepsilon=0.1$, for the first and second modes of motion, as in Fig. 3.

The function $f(s)$ is reasonably simple in both equations (5.3) and (5.6), that is, for shallow or infinite outer depths, and in both of these cases there is no difficulty in evaluating both $f(s)$ and $f^{\prime}(s)$ exactly for arbitrary complex $s=s_{1}+i s_{2}$. The Newton iteration converges rapidly to results shown in Figs. 3-6 and discussed below.

However, for "finite" outer depths, the function $f(s)$ in (5.4) is quite complicated, and for the present purpose an approximate procedure was used. In particular, we a void the necessity of computing the function $\mathrm{I}(H)$ from the integral (4.6) for complex $H$ by writing

$$
\mathbf{I}(H)=\mathbf{I}(|H|)+\left\{\begin{array}{l}
0, \quad|H|<1, \\
i \arg (H), \quad|H|>1,
\end{array}\right.
$$

where the real function $\mathbf{I}(|H|)$ is as in Fig. 2. This is a somewhat ad hoc approximation, which is accurate in both limits $|H| \rightarrow 0, \infty$, and may be expected to model at least the real part of I reasonably well for intermediate $|H|$. It should also be noted that we expect the damping to be small, which means that $s, K$ and $H$ are all "nearly" real, a limit in which again (5.8) is accurate.

We also have some difficulty in estimating the derivative $d \mathbf{l} / d H$ for use in $f^{\prime}(s)$, and hence abandon the exact Newton procedure (5.7) when using (5.4) for $f(s)$, instead estimating $f^{\prime}(s)$ from differences of $f$ for successive iterates. At the same time, in view of (5.5), it is more convenient to choose $K$ rather than $s$ as the independent variable. 


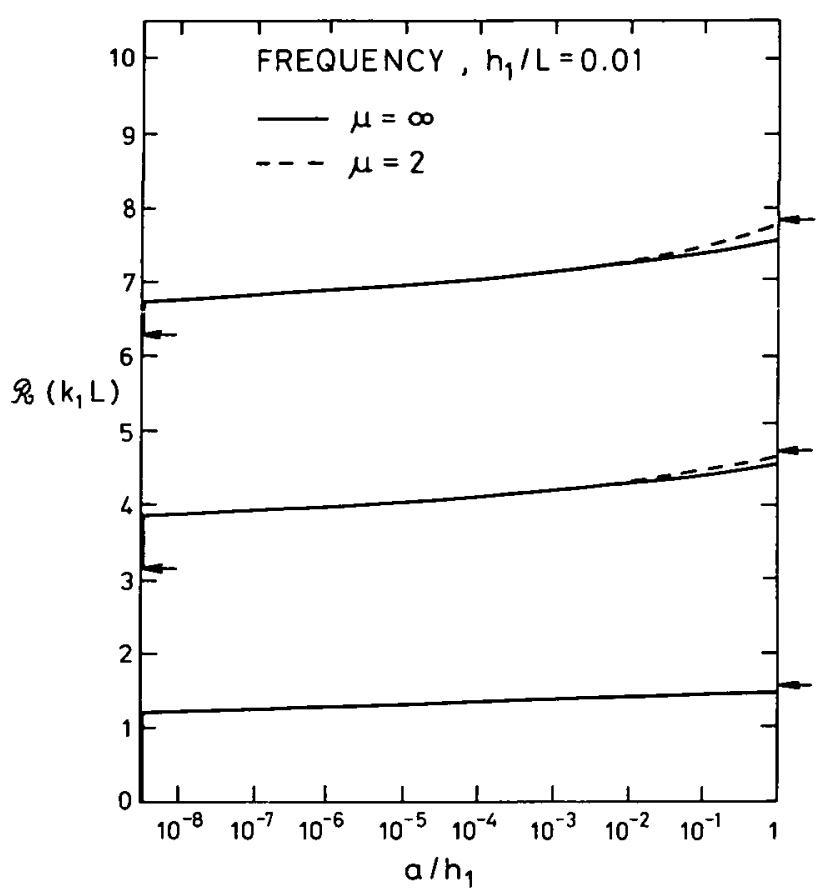

Fig. 5. Real part of frequency for the extremely shallow basin with $\varepsilon=0.01$. Dashed curve is for $\mu=2$, solid curve for $\mu=\infty$. Three modes are shown; $\mu=2, \infty$ are indistinguishable for mode 1 . Note logarithmic scale for the relative gap size $a / h$.

With these modifications, the iteration procedure converges adequately, though less rapidly than for (5.3) or (5.6), and gives results in agreement with the exact theories where overlap is expected.

The ranges of validity of the various formulae may be estimated as follows. All results can only be accurate so long as the inner depth is shallow, that is, using as a rough criterion that the wavelength exceed 10 times the depth, and ignoring damping, when

$$
\varepsilon s_{1}<0.6
$$

For example, if $\varepsilon=0.1$, only the first two modes can be described by the present theory, the third mode with $s_{1} \approx 5 \pi / 2$ being too high a frequency, such that finitedepth effects must be felt on the shelf. On the other hand, the much shallower shelf $\varepsilon=0.01$ allows about 20 shallow-water modes on the shelf.

When (5.9) is satisfied, we still must choose between (5.3) and (5.4). The outer depth is shallow if $K<0.6$, that is, $H<0.3$, or

$$
\varepsilon s_{1}<0.6 \mu^{-\frac{1}{t}} \text {. }
$$




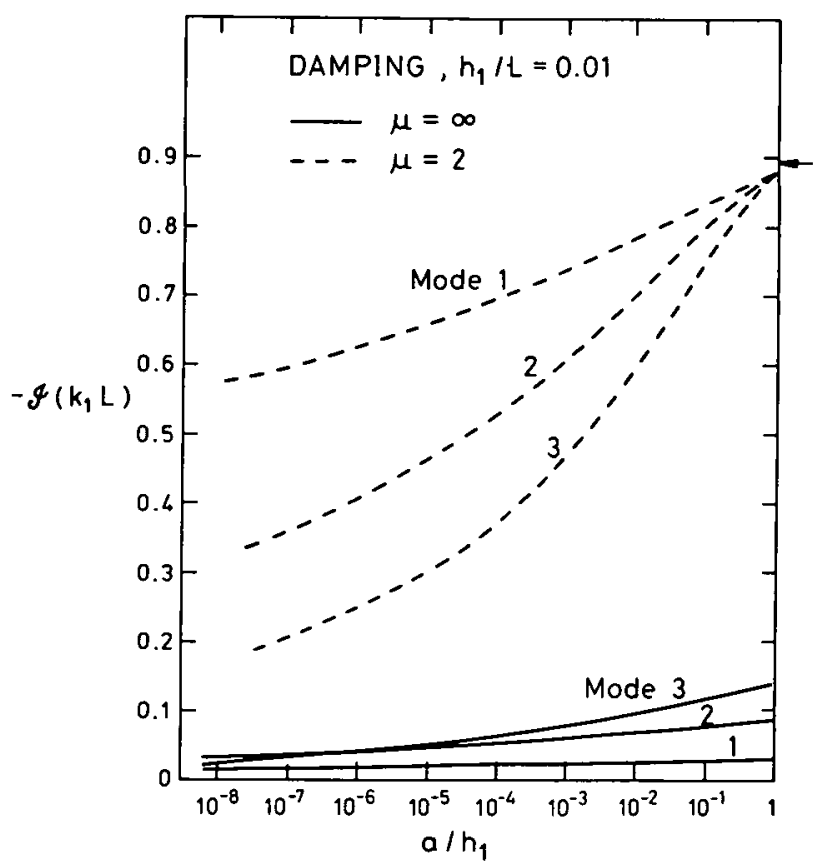

Fig. 6. Damping results corresponding to the frequencies in Fig. 5.

This is only a slight extra imposition if $\mu$ is not large; for example, at $\varepsilon=0.1$ the first mode is shallow up to $\mu=16$, but the second mode only up to about $\mu=1.8$. When $\varepsilon=0.01$, these figures are of course multiplied by 100 , so that there is then virtually no practical limitation on the validity of (5.3).

The range of validity of the finite-outer-depth theory (5.4) is somewhat harder to establish. This theory certainly assumes that $\mu$ is large, and therefore one might expect poor accuracy for $\mu<10$, say. However, Fig. 11 suggests it retains accuracy for smaller values of $\mu$, especially as $a / h_{1} \rightarrow 0$. An indication of this is that in this small-gap limit, the equation (A24) connecting $C$ and $C_{\infty}$ is obeyed exactly by the limiting expressions (A16) and (A25), irrespective of the value of $\mu$. At the other extreme, when $\mu$ is large, for example, $\mu>\left(\varepsilon s_{1}\right)^{-1}$, the infinite-depth theory (5.6) may be used.

Figures 3 and 4 give computed plots against $a_{1} / h$ of the real and imaginary parts of $s$, respectively, for $\varepsilon=0.1$. Only $\mu=2$ and $\mu=\infty$ are shown, and only the first two modes, as anticipated above. The infinite depth results agree to plotting accuracy with computations for $\mu>100$. For values of $\mu \approx 10$, the first-mode frequencies lie between those for $\mu=2$ and $\infty$, and results computed by (5.3) and (5.4) are in close agreement.

The case $\mu=2$ is used because it lies near the borderline of validity of both theories, especially for the second mode. The discrepancy between the dashed and 
solid curves is a measure of the accuracy of the method as a whole for such borderline cases and it is not easy to specify which is the more accurate estimate, especially since (5.4) is not at present being solved without approximation. Fortunately, the general conclusion is that the natural frequencies depend only weakly on $\mu$. Although the damping results vary more with $\mu$ than do the frequencies, so long as $a / h_{1}<0.5$ there is still better than $10 \%$ agreement between the two second-mode damping estimates at $\varepsilon=0.1, \mu=2$.

The frequency results confirm the qualitative predictions of Section 2 . Thus each curve interpolates between the closed-basin frequencies (2.16) at $a / h_{1}=0$ and values at $a / h_{1}=1$ which are close to the open-basin frequencies (2.17). For the lowest mode, however, we must include zero as a "closed" basin frequency. This corresponds to a Helmholtz or pumping mode (as in [3]), in which the water on the shelf is spatially uniform in height, and acts as an energy storage. The limiting frequency is then determined by the relative inertia of the water moving through the small gap, and vanishes as the gap size goes to zero. As the gap increases in size, this Helmholtz mode transforms itself eventually into the lowest (quarter-wave) openbasin mode, of frequency $k_{1} L \approx \pi / 2$. Similarly, each closed-basin natural mode deforms continuously as $a / h_{1}$ increases into the next higher open-basin mode. The dependence of frequency on gap size is continuous but rapid near $a / h_{1}$, as anticipated, and only for very small gaps indeed is the basin effectively closed.

This is shown even more dramatically by Figs 5 and 6 for the much shallower basin with $\varepsilon=0.01$. In this case we have chosen a logarithmic scale for $a / h_{1}$ since, on a linear scale, the "action" takes place too close to $a / h_{1}=0$ to be plotted. Three modes are shown, with $\mu=2$ and $\mu=\infty$, there being now no doubt about the accuracy of the respective determining equations (5.3) and (5.6).

The results in Figs 4 and 6 for the imaginary part of $s$, that is, for the damping, exhibit a much greater variation with $a / h_{1}$ than do the frequency results. Obviously the damping always tends to zero as $a / h_{1} \rightarrow 0$, and it also does so ultimately like the inverse logarithm of $a / h_{1}$, but the real reduction in damping from the open-basin value is significant even at moderate values of $a / h_{1}$.

The open-basin value given by (3.13) is 0.891 at $\mu=2$, and this value is closely approximated by all three modes at $a / h_{1}=1$, for the shallow basin $\varepsilon=0.01$ of Fig. 6 , if not quite so closely at $\varepsilon=0.1$ in Fig. 4 . Note that the open-basin value (3.13) is identical for all modes, a result confirmed by Fig. 6 at $a / h_{1}=1$.

As $a / h_{1}$ decreases from unity, the damping curves become quite different for different modes. In this respect there is an interesting difference between the shallowwater and deep-water results. When the outer depth is large, the shorter waves radiate energy to infinity more easily and hence are the more strongly damped. Thus the lowest-order mode must predominate. Conversely, when the water is shallow, it appears that the shorter waves are less damped than the longer waves, and therefore one should expect higher modes to predominate. This tendency is greatest for the 
lowest mode as $a / h_{1} \rightarrow 0$, indicating that the Helmholtz mode is relatively strongly damped in that limit.

Although the results shown in Figs 3-6 indicate a transition from closed to nearlyopen basins as $a / h_{1}$ increases from zero, it should be noted that a truly open basin is never achievable by variation of $a / h_{1}$. Thus, although the frequencies are closer to the values (2.17) at $a=h_{1}$ than for any other value of $a$, the basin without a wall is still not quite fully open.

This observation is even more important for the damping, which would be zero for a fully-open basin, but is never zero at any fixed $a>0$. Indeed, generally the damping as measured by $-\mathscr{I} m\left(k_{1} L\right)$ appears to take its maximum value when the wall is absent. The general order of magnitude of the damping is given by (3.13) if $\mu$ is finite, irrespective of $\varepsilon$, but tends to zero as $\varepsilon \rightarrow 0$ for infinte $\mu$. Note, for example, the dramatic decrease in the damping at $\mu=\infty$ from Fig. 4 to Fig. 6 . Thus the only limit in which the true open-basin results are attained is that for infinite outer depth, as the shallowness of the inner basin tends to zero.

All computations in this paper were performed on a TRS-80 micro-computer.

\section{Acknowledgement}

Critical comments and suggestions by Dr. H. Allison are gratefully acknowledged.

\section{Appendix A: Blockage coefficient of a depth change with a wall}

Consider the conformal mapping illustrated in Fig. 7 from the $z$-plane to the $t$ plane, where

$$
z=\frac{h_{1}}{\pi} \log \left[t^{\mu}\left(\frac{t+\beta}{1+\beta t}\right)\right]-i h_{2} .
$$

The left- and right-side depths are $h_{1}$ and $h_{2}$, respectively, and $\mu=h_{2} / h_{1}$. The parameter $\beta$ controls the wall height, and we require

$$
1 \leqslant \beta \leqslant\left|\frac{\mu+1}{\mu-1}\right| \text {. }
$$

We shall find that, as $\beta \downarrow 1$, the wall closes off the gap entirely whereas, as $\beta \uparrow|(\mu+1) /(\mu-1)|$, the wall disappears. The logarithm function is defined by its principal branch such that, for $t=|t| e^{i \arg (t)}, \log t=\log |t|+i \arg (t)$, with $-\pi<\arg (t) \leqslant \pi$.

The validity of this mapping may be checked as follows. Along $F G$ we have $t$ real and $t>1$. Then, clearly, the logarithm is real and we have 

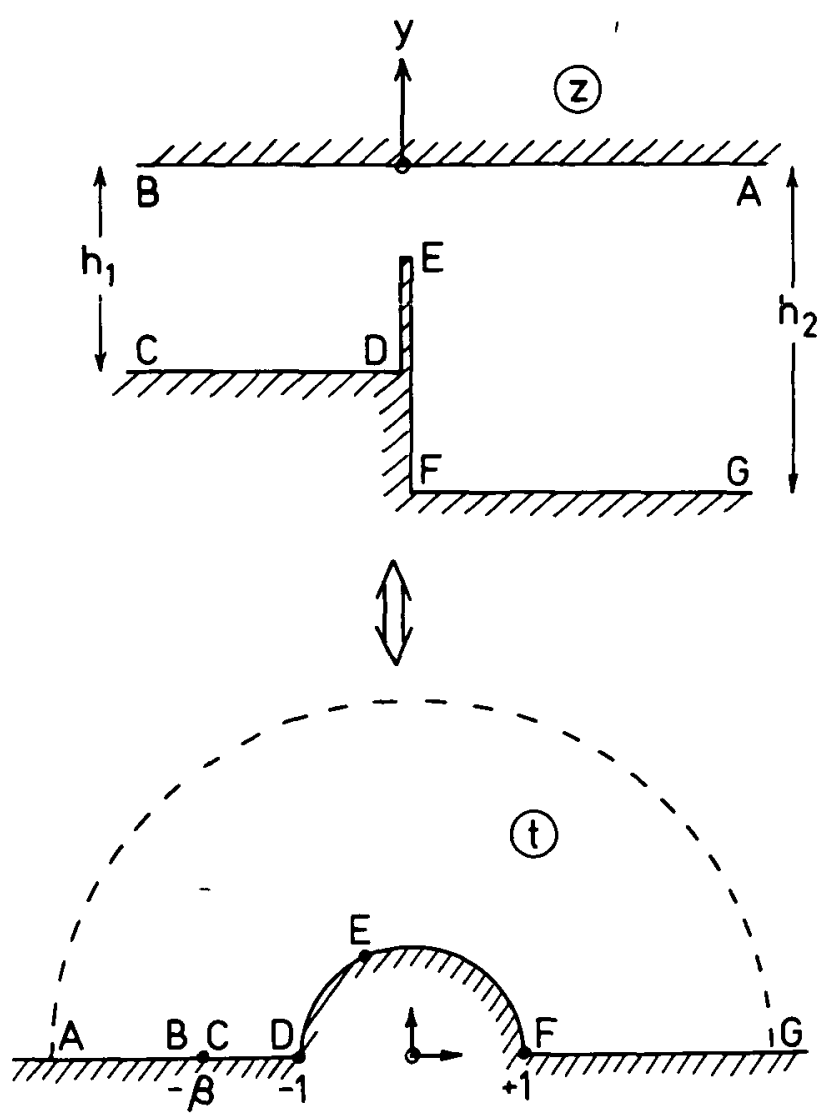

Fig. 7. Sketch of conformal mapping of transition region in the complex z-plane between depth $h_{1}$ and depth $h_{2}$ onto the exterior of the unit semicircle in the complex $t$-plane.

$$
x=\frac{h_{1}}{\pi} \log \left(t^{\mu} \frac{t+\beta}{1+\beta t}\right) \text { and } y=-h_{2} .
$$

We require that $x$ increase monotonically from zero at $F$ to infinity at $G$, which is true so long as $\beta$ satisfies the inequality (A2).

On $D E F$, the unit semi-circle $t=e^{i \theta}, 0 \leqslant \theta \leqslant \pi$, we have

$$
z=\frac{h_{1}}{\pi} \log e^{i(\mu-1) \theta}\left(\frac{e^{i \theta}+\beta}{e^{-i \theta}+\beta}\right)-i h_{2},
$$

that is,

$$
x=0 \text { and } y=h_{1} Y(\theta)
$$

where

$$
Y(\theta)=\frac{\theta}{\pi}(\mu-1)-\mu+\frac{2}{\pi} \arctan \left(\frac{\sin \theta}{\cos \theta+\beta}\right) .
$$


The points $F$ and $D$ correspond to $\theta=0, \pi$, respectively, while the point $E$ at the top of the wall corresponds to the maximum of $Y(\theta)$, that is, to $\theta=\theta_{m}$ where $Y^{\prime}\left(\theta_{m}\right)=0$, satisfying

$$
\cos \theta_{m}=-\frac{\mu+1+(\mu-1) \beta^{2}}{2 \mu \beta} .
$$

Thus the gap size is given by

$$
\frac{a}{h_{1}}=-Y\left(\theta_{m}\right)
$$

On the segment $C D,-\beta<t<-1$, we have as required that $y=-h_{1}$ and $x<0$ if (A2) holds, and similarly on $A B, t<-\beta$, we have $y=0$ and $x$ ranges from $-\infty$ at $t=-\beta$ to $+\infty$ at $t=-\infty$. This completes verification of boundary mappings, and spot checks of interior points confirm the validity of the complete conformal map.

We are particularly concerned with the properties of this mapping as $t \rightarrow-\beta, B C$, and $|t| \rightarrow \infty, A G$. Thus, as $t \rightarrow-\beta$,

that is,

$$
z \rightarrow-i h_{1}+\frac{h_{1}}{\pi} \log \left|\frac{\beta^{\mu-1}}{\beta-\beta^{-1}}\right|+\frac{h_{1}}{\pi} \log (t+\beta),
$$

$$
\frac{1}{\pi} \log |t+\beta| \rightarrow \frac{x}{h_{1}}-\frac{1}{\pi} \log \left|\frac{\beta^{\mu-1}}{\beta-\beta^{-1}}\right| .
$$

Similarly, as $|t| \rightarrow \infty$,

$$
z \rightarrow \frac{h_{1}}{\pi} \log \frac{\mathrm{t}^{\mu}}{\beta}-i h_{2}
$$

that is,

$$
\frac{1}{\pi} \log |t| \rightarrow \frac{x}{h_{2}}+\frac{1}{\pi \mu} \log \beta .
$$

We now wish to solve the canonical flow problem of Fig. 8 in which a uniform stream with unit total flux approaches from the left and emerges on the right as a corresponding unit-flux stream. Thus the velocity potential is required to satisfy

$$
\phi \rightarrow \begin{cases}\frac{x}{h_{1}}-C, & x \rightarrow-\infty, \\ \frac{x}{h_{2}}+C, & x \rightarrow+\infty .\end{cases}
$$

The constant $C$ in (A10) is the blockage coefficient of the depth transition, which we aim to determine.

Now the corresponding flow in the $t$-plane must be that for a source at $(-\beta, 0)$ outside the unit circle, and we can write down the complete potential $f=\phi+i \psi$ for that flow by Milne-Thomson's circle theorem. Hence 


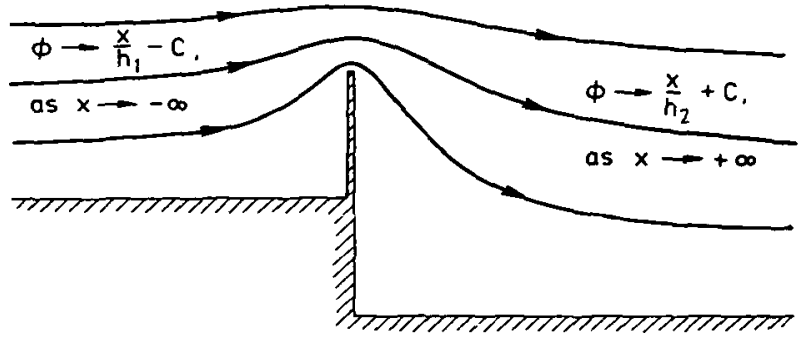

Fig. 8. Sample of streamlines through the transition region from depth $h_{1}$ to depth $h_{2}$.

$$
f=\frac{1}{\pi} \log (t+\beta)+\frac{1}{\pi} \log \left(t^{-1}+\beta\right)+K,
$$

for some constant $K$, which can be taken as real. Now, as $t \rightarrow-\beta$, we have

$$
\phi \rightarrow \frac{1}{\pi} \log |t+\beta|+\frac{1}{\pi} \log \left(\beta-\beta^{-1}\right)+K
$$

or, using (A8),

$$
\phi \rightarrow \frac{x}{h_{1}}-C
$$

where

$$
C=-K+\frac{\mu-1}{\pi} \log \beta-\frac{2}{\pi} \log \left(\beta-\beta^{-1}\right)
$$

Similarly, as $|t| \rightarrow \infty$,

$$
\begin{aligned}
\phi & \rightarrow \frac{1}{\pi} \log |t|+\frac{1}{\pi} \log \beta+K \\
& \rightarrow \frac{x}{h_{2}}+C,
\end{aligned}
$$

where

$$
C=K+\frac{\mu+1}{\pi \mu} \log \beta .
$$

Upon elimination of $K$ between equations (A12) and (A13) we have as our formula for the blockage coefficient

$$
2 \pi C=\left(\mu+\mu^{-1}\right) \log \beta-2 \log \left(\beta-\beta^{-1}\right) .
$$

In the absence of the wall, that is, when $\beta=|(\mu+1) /(\mu-1)|$, this agrees with the result of Tuck [7], namely 


$$
2 \pi C=\left(\mu+\mu^{-1}\right) \log \left|\frac{\mu+1}{\mu-1}\right|-2 \log \left|\frac{4 \mu}{\mu^{2}-1}\right| .
$$

We are particularly interested in the small-gap limit $\beta \downarrow 1$, in which case

$$
2 \pi C \rightarrow-2 \log 2(\beta-1) \text {. }
$$

This can be expressed in terms of the actual gap size by noting from (A6) that, as

$$
\beta \downarrow 1, \quad \theta_{m} \rightarrow \pi-\sqrt{ }[(2 / \mu)(\beta-1)]
$$

and hence, from (A5) and (A7),

$$
\frac{a}{h_{1}} \rightarrow \frac{2}{\pi} \sqrt{ }(2 \mu(\beta-1))
$$

Thus, as $a / h_{1} \rightarrow 0$,

$$
C \rightarrow \frac{2}{\pi} \log \left(\frac{2}{\pi a} \sqrt{ }\left(h_{1} h_{2}\right)\right),
$$

a result that can also be obtained by matching (see Tuck [6]). Figure 9 shows a plot of $C$ against $a / h_{1}$ for various values of $\mu$, the exact result 4) being the solid curve and the small-gap asymptote the dashed curve.

We are also interested in the limit as $\mu \rightarrow \infty$, that is, as the right-hand depth $h_{2}$ becomes large. The inequality (A2) then demands that $\beta<1+(2 / \mu) \rightarrow 1$. It is convenient to set

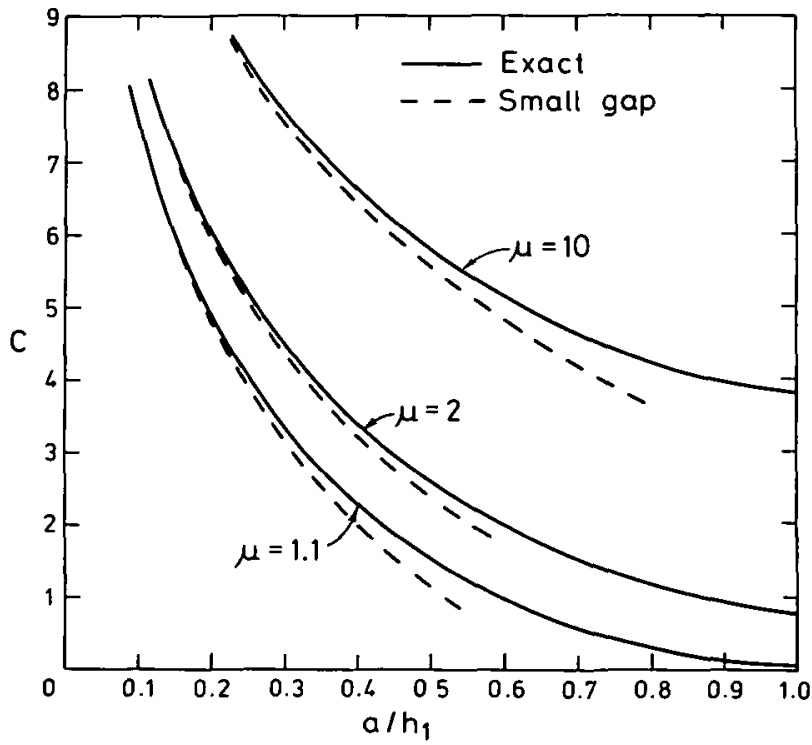

Fig. 9. Blockage coefficient $C$ as a function of $a / h_{1}$ for various values of $\mu$. Dashed curves are small-gap approximations. 


$$
\beta=1+\frac{2}{\mu} \sin ^{2} \alpha
$$

and hold $\alpha$ fixed as $\mu \rightarrow \infty$. Then we find from (A6) that $\theta_{m} \rightarrow \pi-(1 / \mu) \sin 2 \alpha$ and hence from (A7) and (A5) that

$$
\frac{\pi \mathrm{a}}{h_{1}}=2 \alpha+\sin 2 \alpha,
$$

so that the gap size $a$ increases monotonically from 0 to $h_{1}$ as $\alpha$ goes from 0 to $\pi / 2$.

On the other hand, the actual blockage coefficient $C$ becomes infinite on the limit as $\mu \rightarrow \infty$ and $\beta \rightarrow 1$, with

$$
2 \pi C \rightarrow-2 \log 2(\beta-1)+\mu(\beta-1) .
$$

In fact, in this limit the blockage coefficient $C$ defined as in Fig. 8 is of less interest than a coefficient $C_{\infty}$ defined by Fig. 10 which measures the finite potential jump between the uniform stream at $x=-\infty$ and the source-like flow into the quarterplane at $x=+\infty$. Although we can determine $C_{\infty}$ by an entirely separate conformal mapping, matching procedures enable a limiting relationship to be established between $C_{\infty}$ and $C$ as follows.

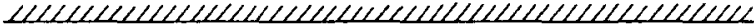

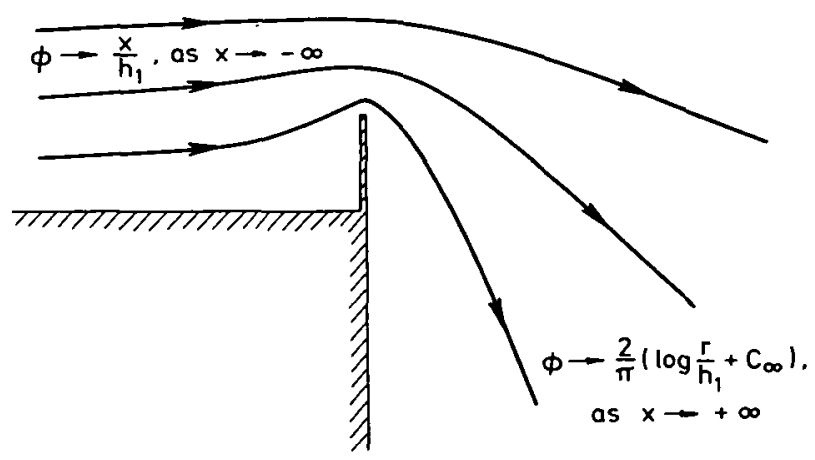

Fig. 10. Sample streamlines through the transition region from depth $h_{1}$ to infinite depth.

Thus, as $a / h_{2} \rightarrow 0$, the flow in $x>0$ tends to that induced by a source of strength $\frac{1}{4}$ at the origin, in a channel of depth $h_{2}$, having the complex potential

$$
f(z)=\frac{2}{\pi} \log 2 \sinh \frac{\pi z}{2 h_{2}}+2 C .
$$

In the limit as $x / h_{2} \rightarrow \infty$, we have

$$
\phi \rightarrow \frac{x}{h_{2}}+2 C \text { as } x \rightarrow+\infty,
$$


so that we must add the constant $C$ to the complete potential defined in Fig. 8; hence we now have

$$
\phi \rightarrow \frac{x}{h_{1}} \text { as } x \rightarrow-\infty .
$$

Now for $r=|z| \ll h_{2}$ in (A20) we have

$$
\begin{aligned}
\phi & \rightarrow \frac{2}{\pi} \log \frac{\pi r}{h_{2}}+2 C \\
& =\frac{2}{\pi}\left(\log \frac{r}{h_{1}}+C_{\infty}\right),
\end{aligned}
$$

where

$$
\begin{aligned}
C_{\infty} & =\frac{\pi}{2}\left(2 C-\frac{2}{\pi} \log \frac{\mu}{\pi}\right) \\
& =-\frac{2}{\pi} \log \frac{2}{\pi} \mu(\beta-1)+\frac{1}{\pi} \mu(\beta-1) \\
& =\sin ^{2} \alpha-\log \frac{4}{\pi} \sin ^{2} \alpha,
\end{aligned}
$$

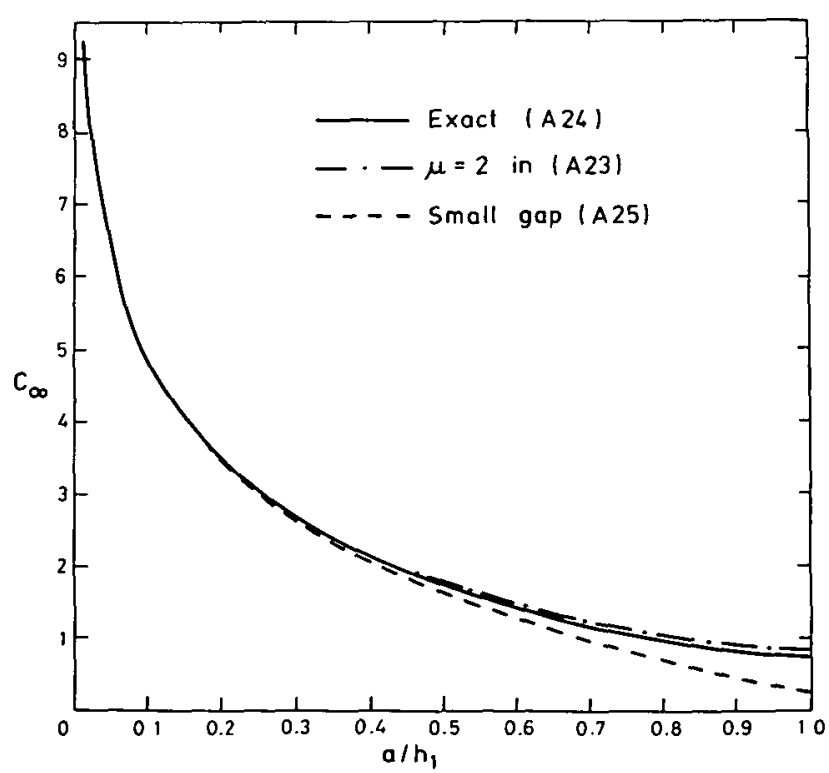

Fig. 11. Coefficient $C_{\infty}$ for flow of Fig. 10 as a function of $a / h_{1}$. The exact result is the solid curve and the small-gap approximation is the dashed curve. The chain-dotted curve is the value estimated from the finite-depth coefficient $C$ as in Fig. 9, using $\mu=2$. 
which is a finite quantity, depending on the depth ratio $a / h_{1}$ through the parametric equation (A 18). Figure 11 shows $C_{\infty}$ as a function of $a / h_{1}$, together with (dashed) its small-gap limit as $\alpha \rightarrow 0$, that is,

$$
C_{\infty} \rightarrow \log \frac{4}{\pi} \frac{h_{1}^{2}}{a^{2}} \quad \text { as } \frac{a}{h_{1}} \rightarrow 0,
$$

and (chain dotted) the resuit of setting $\mu=2$ in (A23), using the exact result (A14) for $C$ at $\mu=2$. The closeness of the last result to the exact $C_{\infty}$ indicates that the infinite- $\mu$ limit is still very accurate down to $\mu$ values as low as 2 .

\section{References}

[1] H. Allison and A. Grassia, "Sporadic sea-level oscillations along the Western Australian coastline", to be published in Austral. J. Mar. Freshwater Res. 30 (1979).

[2] C. Macaskill, "Reflexion of water waves by a permeable barrier", J. Fluid Mech. 95(1979), 141-157.

[3] J. W. Miles, "Resonant response of harbors (the harbor paradox revisited)", Proc. 8th Symp. Naval Hydro. (1970), 95-115.

[4] V.J. Monacella, "On ignoring the singularity in the numerical evaluation of Cauchy principal value integrals", David Taylor Model Basin, Report 2356 (1967).

[5] E. O. Tuck, "Shallow-water flows past slender bodies", J. Fluid Mech. 26 (1966), 81-95.

[6] E. O. Tuck, "Matching problems involving flow through small holes", Adv. in Appl. Mech. 15 (1975), 89-158.

[7] E. O. Tuck, "Some classical water-wave problems in variable depth", in Proc. I.U.T.A.M. Symp. Waves on water of variable depth (ed. D. G. Provis and R. Radok) (Springer-Verlag, Berlin, 1976), pp. 9-20.

[8] E. O. Tuck, H. Allison, S. Field and J. Smith, "The effect of a submerged reef chain on periods of sealevel oscillations in Western Australia", to be published in Austral.J. Mar. Freshwater Res. 31 (1980).

[9] J. V. Wehausen and E. V. Laitone, "Surface waves", in Handbuch der Physik, Vol. 9 (ed. S. Flugge), (Springer-Verlag, Berlin, 1960), pp. 446-778.

Department of Applied Mathematics

University of Adelaide

Adelaide

South Australia 5000 